\title{
Obstacle Avoidance of Robotic Formations Based on Fluid Mechanical Modeling
}

\author{
Rabie Soukieh, Iman Shames and Barış Fidan
}

\begin{abstract}
This paper is on obstacle avoidance of swarms of robots moving in certain geometric planar formations. Focus is given to a particular obstacle avoidance approach, which is based on the fluid mechanical principle known as the Circle Theorem. Considering the motion region as a fictitious fluid environment surrounding the obstacles, fluid streamlines are calculated which correspond to unique smooth paths that a robot or a robotic formation can follow without colliding with the obstacles. The design and analysis are initially performed assuming simple integrator dynamics for each agent, and later extended for more realistic non-holonomic unicycle dynamic agent models, with the help of proportional integral (PI) control. The fluid dynamics based designs developed for obstacle avoiding motion control of agents, moving in a prescribed rigid formation are novel, and successfully tested via an extensive set of simulations. Application of the developed designs for motion control of unmanned aerial vehicle (UAV) formations under the constraint of constant speed is also presented.
\end{abstract}

\section{INTRODUCTION}

Formation control of multi-agent systems continues to be an expanding area of research, an important part of which is to provide obstacle avoidance capabilities for the formation of mobile agents. Some of the formation control techniques already presented in the literature can be seen in, [1], [2], [3], [4]. A specific area of interest in producing collision-free paths for multi-agent formations, is to use concepts from fluid mechanics. There is little coverage of such applications in the literature: one approach has been suggested in [5] based on the Circle Theorem, and more recently by the use of Panel Methods in [6], [7]. The fluid mechanics methods are centered around the use of stream functions and streamlines, which form part of the potential flow solution and are free from local minima. The streamlines are determined analytically by using complex potentials of flows such as uniform flow, vortex (or source) to represent obstacles (with radius $R_{o i}$ ) and the goal is represented by sinks. In this paper, we apply the Circle Theorem and its extensions to find collision-free streamline paths, which formations can follow.

A multi-agent formation $F$ is a collection of mobile robot agents $A_{1}, \ldots, A_{m}$. It is represented by an underlying graph $G_{F}=\left(V_{F}, E_{F}\right)$ with a vertex set $V_{F}$ and an edge set $E_{F}$, [8]. Each agent $A_{i}$ is represented by a vertex $i \in$ $V_{F}$, and pairs of neighboring agents $\left(A_{i}, A_{j}\right)$ by a directed

This work is supported by NICTA, which is funded by the Australian Government as represented by the Department of Broadband, Communications and the Digital Economy and the Australian Research Council through the ICT Centre of Excellence program.

Rabie Soukieh is with the Australian National University u4113369@anu.edu.au, Iman Shames and Barış Fidan are with Australian National University and National ICT Australia \{Iman.Shames, Baris.Fidan\}@anu.edu.au edge $\overrightarrow{(i, j)} \in E_{F}$ which represents a sensing, control or communication link between agents $A_{i}$ and $A_{j}$. The directed edge $\overrightarrow{(i, j)}$ indicates that $A_{i}$ follows $A_{j}$, and must maintain a desired distance $d_{i j}$, from $A_{j}$. In our work a graph with unidirectional edges is termed an asymmetric graph where only one agent maintains $d_{i j}$ in an edge (as above), while one with bidirectional edges is a symmetric graph and both agents in an edge maintain $d_{i j}$.

The number of directed edges that originate from a vertex in a directed underlying graph, equals the number of distance constraints it must keep with other agents. In addition, an agent that has no directed edge originating from it is called a leader (which has 2 degrees of freedom (DOF)) and can move freely in $\Re^{2}$. If only one directed edge originates from it, it is called the first follower (or a co-leader with 1-DOF) and it can only rotate around the agent it follows in order to meet its distance constraint. If two or more directed edges originate from it, the agent is called an ordinary follower (which has 0 DOF) and its motion completely depends on the agents it follows. As detailed in [8], [9], the sum of DOFs of individual agents in $\Re^{2}$ for any of these formations is at most 3 , i.e. two for translation and one for rotation.

We say a formation has a leader-follower structure if, there is exactly one agent with 2 DOFs, and one agent with 1 DOF. The rest of the agents have 0 DOF. An example for such a formation is depicted in figure 3 , where $A_{1}$ is the leader and $A_{2}$ is the first follower.

A rigid formation is one in which the distance $d_{i j}$ between each neighboring agent pair $\left(A_{i}, A_{j}\right)$ remains constant, which implicitly means that the formation shape as well as the remaining inter-agent distances, are maintained during any continuous motion of the formation. If every agent in the formation satisfies all of the constraints on it, after having satisfied other constraints within the formation, then the formation is called constraint-consistent. Furthermore, a formation that is both rigid and constraint-consistent is called persistent. Thorough definitions and analysis of rigidity, constraint-consistence and persistence can be found in [8], [9].

This paper uses the distributed control principles and techniques and the rigid graph theory based formations presented in [10], [11]. More details on formation control graphs and underlying graphs can be found in [8], [9]. We focus on the leader-follower hierarchical sensing and control structure and the application of a set of fluid mechanical concepts for obstacle avoidance of formations. Noting that earlier studies consider motion of point-agent modeled robots with 


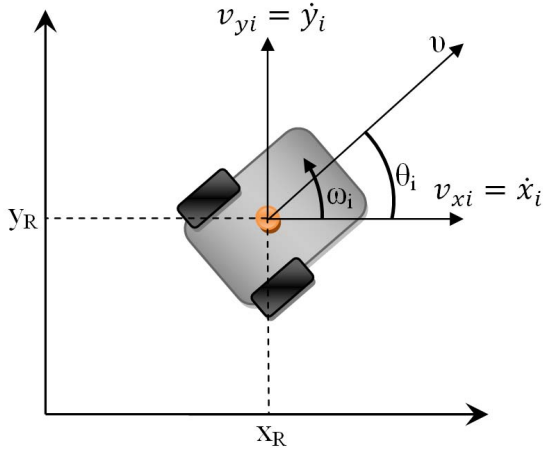

Fig. 1. Model assumed for agents

single velocity integrator dynamic model and they do not focus on formation control, our main contributions are: Fluid dynamics based design and analysis of obstacle-avoiding motion control of swarms of agents moving in rigid formation, where the agents have (a) limited but not necessarily constant speeds, (b) non-holonomic unicycle dynamics with constant speed constraint, i.e. Dubins' vehicle dynamics, noting that the latter case well applies to UAV formations.

\section{PRoblem Definition}

\section{A. Agent Modeling}

Consider agents to be points in a 2-dimensional Cartesian environment $\left(\Re^{2}\right)$. As a starting point, simple-single velocity integrator dynamics are assumed for agents, as described by

$$
\begin{gathered}
\dot{p}_{i}(t)=v_{i}(t) \\
\left\|v_{i}(t)\right\|=\bar{v}_{i}
\end{gathered}
$$

where $p_{i}(t)=\left[x_{i}(t), y_{i}(t)\right]^{T}, v_{i}(t)=\left[v_{x i}(t), v_{y i}(t)\right]^{T} \in \Re^{2}$ denote, respectively, the position and velocity of the agent $A_{i}$ at time $t$. The agent dynamics is then extended to the non-holonomic unicycle model

$$
\begin{aligned}
& \dot{x}_{i}(t)=v_{x i}(t)=\bar{v}_{i} \cos \theta_{i}(t) \\
& \dot{y}_{i}(t)=v_{y i}(t)=\bar{v}_{i} \sin \theta_{i}(t) \\
& \dot{\theta}_{i}(t)=\omega_{i}(t)
\end{aligned}
$$

where $\dot{x}_{i}(t)$ and $\dot{y}_{i}(t)$ are the velocity components in the $x y$ plane, $\theta_{i}(t)$ is the heading, $\bar{v}_{i}$ is the translational speed and $\omega_{i}(t)$ is the angular velocity of $A_{i}$, as illustrated in Figure 1, where $\left[x_{R}, y_{R}\right]^{T}$ is the starting location. It is assumed here that the agent knows its current position and can calculate its heading $\theta_{i}(t)$ at any time $t$.

\section{B. Formations Modeled as Ellipse}

In this paper, we consider formations as ellipses, where this ellipse is the minimum volume ellipse that surrounds all the agents in the formation. An ellipse, with a semi-minor axis length $b_{m}$ and a semi-major axis length $a_{m}$, is defined parametrically by

$$
\begin{aligned}
& x=e_{1}+a_{m} \cos \theta \\
& y=e_{2}+b_{m} \sin \theta \\
& -\pi \leq \theta \leq \pi
\end{aligned}
$$

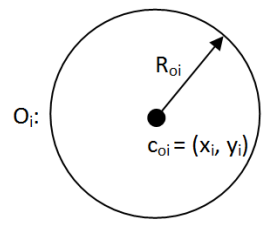

Fig. 2. Obstacles modeled as circular discs

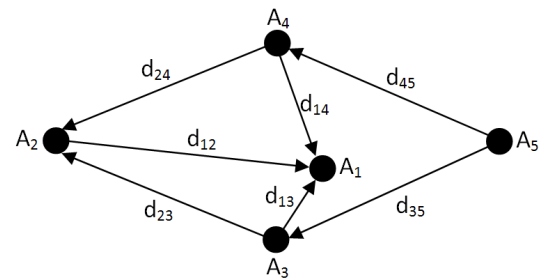

Fig. 3. Directed underlying graph of a persistent formation, with a leaderfollower structure

where $\left[e_{1}, e_{2}\right]^{T}$ is the centre of the ellipse. The motion of the ellipse formation will be constrained by the ellipse dimensions $a_{m}$ and $b_{m}$, i.e. the formation will only be able to move in places which are clear from obstacles by at least a distance $b_{m}$ (and in some regions $a_{m}$ ) from the centre of the ellipse.

\section{Obstacle Modeling}

For the rest of this paper, each obstacle has been assumed to be static (not moving) and in the form of a circular disc, see Figure 2. Obstacle $i$ is denoted by $O_{i}=C\left(c_{o i}, R_{o i}\right)$ where $c_{o i}$ is the centre and $R_{o i}$ is the radius of obstacle $i$, for $i=1, \ldots, N$. Each agent can measure $c_{o i}$ and $R_{o i}$ all the time for $i=1, \ldots, N$. If the initial agent position is $p(0)=p_{0}$ and the target position is $p_{f}$, we want to generate $v(t)$ such that there is a time $t_{f}>0$ where, $p\left(t_{f}\right)=p_{f}$ and $p(t) \cap O_{i}=\emptyset, \forall i=1, \ldots, N$ and $\forall 0 \leq t \leq t_{f}$. This means that we want our path to be free from obstacles and the position of the agent at the final time corresponds to the goal position.

\section{Multi-Agent Systems, Formation Control and Obstacle Avoidance}

In this paper we deal with the hierarchical leader-follower formation depicted in Figure 3. The particular problem which we address for this formation is outlined below.

Problem 1: Formation Control with Obstacle Avoidance. Consider a swarm $F$ of robot agents $A_{1}, \ldots, A_{m}, m \geq 3$ moving in a persistent formation, defined by a structure e.g. the one depicted in Figure 3 and a set of desired interagent distances $d_{i j}$, between neighbor agent pairs $\left(A_{i}, A_{j}\right)$. Assume that the initial positions $p_{i 0}$ of agents $A_{i}, \ldots, A_{j}$ are known and are consistent with the distance constraints $d_{i j}$. The motion control task is to move $F$ to a final desired position and orientation, defined by a set of individual final positions $p_{i f}$ of $A_{i}$, where $p_{i f}$ satisfy the distance 
constraints $d_{i j}$, cohesively, while avoiding collision with an arbitrary number $N$ of obstacles $O_{1}, \ldots, O_{N}$. The task needs to be performed using a decentralized control scheme and individual controllers of the agents $A_{1}, \ldots, A_{m}$.

Here cohesively means that the shape of $F$ must not deform and the distance constraints of $F$ during motion cannot be violated.

The velocity $v_{i}(t)=\left[v_{x i}(t), v_{y i}(t)\right]^{T}$ is considered as the control signal to be generated by the individual controller of agent $A_{i}$. It is required that $v_{i}(t)$ is continuous and satisfies $\left\|v_{i}(t)\right\| \leq \bar{v}$ for some constant maximum speed limit $\bar{v}>0$ at any $t \geq 0$ for any $i \in 1, \ldots, m$. It is assumed that each agent $A_{i}$ knows its final desired position $p_{i f}$ and can sense its own position $p_{i}(t)$ and velocity $v_{i}(t)$ as well as the position $p_{j}(t)$ of each agent $A_{j}$ it follows at any time $t \geq 0$. It is also assumed that the distance sensing range for a neighbor agent pair $\left(A_{i}, A_{j}\right)$ is sufficiently larger than the desired distance $d_{i j}$ to be maintained.

\section{BASE FORMATION CONTROL LAWS}

The control laws which follow are derived in [8] from basic vector analysis and ideas from the virtual vector field concept. In this approach, the aim is to obtain the overall velocity vector for each $A_{i}$, as the superposition of the vectors defining each of the separate motion tasks for $A_{i}$. In this case, these two motion tasks for $A_{i}$ are, (a) to maintain a distance constraint with each agent $A_{j}$ it follows and (b) to move towards a final destination. Please refer to [8], [10] for a complete analysis and overview of these control laws. Next, we present the individual control laws of the agents for cohesive motions of the formation, when there is no obstacle in the motion space, considering single integrator agent dynamics model (II.1).

\section{A. Control Law for the Ordinary Followers}

Agents $A_{3}, A_{4}$ and $A_{5}$ are ordinary followers in the leaderfollower structure of Figure 3. The control law for these agents is given by

$$
\begin{aligned}
& v_{i}(t)=\bar{v} \beta_{i}(t) \delta_{i d}(t) /\left\|\delta_{i d}(t)\right\| \\
& \delta_{i d}(t)=p_{i d}(t)-p_{i}(t)=\bar{p}_{j k}\left(t, p_{i}(t)\right)-p_{i}(t) \\
& \beta_{i}(t)= \begin{cases}0, & \left\|\delta_{i d}(t)\right\|<\varepsilon_{k} \\
\frac{\left|\delta_{i d}(t)\right|-\varepsilon_{k}}{\varepsilon_{k}} & \varepsilon_{k} \leq\left|\delta_{i d}(t)\right|<2 \varepsilon_{k} \\
1, & \left\|\delta_{i d}(t)\right\| \geq 2 \varepsilon_{k}\end{cases}
\end{aligned}
$$

where $\bar{v}>0$ is the constant maximum speed of the agents and $\varepsilon_{k}>0$ is a small design constant. The term $\delta_{i d}(t)$ is the distance between the desired and actual positions of the ordinary follower agent. Note that $\bar{p}_{j k}\left(t, p_{i}(t)\right)=$ $\left[\bar{x}_{j k}\left(t, p_{i}(t)\right), \bar{y}_{j k}\left(t, p_{i}(t)\right)\right]^{T}$, where $\bar{p}_{j k}\left(t, p_{0}\right)$ for any $p_{0} \in$ $\Re^{2}$ denotes the intersection of the circles $C\left(p_{j}(t), d_{i j}\right)$ and $C\left(p_{k}(t), d_{i k}\right)$ that is closer to $p_{0}$, and in the notion $C(\cdot, \cdot)$ the first argument denotes the center and the second denotes the radius. In (III.1), the switching term $\beta_{i}(t)$ is used to avoid rapid movements due to small but acceptable errors in the desired inter-agent distances.

\section{B. Control Law for the First Follower}

Agent $A_{2}$ is the first follower in Figure 3. The control law for this agent is as follows

$$
\begin{aligned}
& v_{i}(t)=\beta_{i}(t) v_{i 1}(t)+\sqrt{1-\beta_{i}^{2}(t)} v_{i 2}(t) \\
& \delta_{j i}(t)=\left(\delta_{j i x}(t), \delta_{j i y}(t)\right)=p_{j}(t)-p_{i}(t) \\
& \bar{\delta}_{j i}(t)=\left\|\delta_{j i}(t)\right\|-d_{i j} \\
& \beta_{i}(t)= \begin{cases}0, & \left|\bar{\delta}_{j i}(t)\right|<\varepsilon_{k} \\
\frac{\left|\bar{\delta}_{j i}(t)\right|-\varepsilon_{k}}{\varepsilon_{k}} & \varepsilon_{k} \leq\left|\bar{\delta}_{j i}(t)\right|<2 \varepsilon_{k} \\
1, & \left|\bar{\delta}_{j i}(t)\right| \geq 2 \varepsilon_{k}\end{cases}
\end{aligned}
$$

where

$$
\begin{array}{r}
v_{i 1}(t)=\bar{v} \operatorname{sgn}\left(\bar{\delta}_{j i}(t)\right) \delta_{j i}(t)\left\|\delta_{j i}(t)\right\| \\
v_{i 2}(t)=\bar{v} \bar{\beta}_{i}(t) \operatorname{sgn}\left(\delta_{i f}(t)^{T} \bar{\delta}_{j i}^{\perp}(t)\right) \bar{\delta}_{j i}^{\perp}(t) \\
\delta_{i f}(t)=p_{i f}(t)-p_{i}(t) \\
\bar{\delta}_{j i}^{\perp}(t)=\left(-\delta_{j i y}(t), \delta_{j i x}(t)\right) /\left\|\delta_{j i}(t)\right\| \\
\bar{\beta}_{i}(t)= \begin{cases}0, & \left|\delta_{i f}(t)\right|<\varepsilon_{f} \\
\frac{\left|\delta_{i f}(t)\right|-\varepsilon_{f}}{\varepsilon_{f}} & \varepsilon_{f} \leq\left\|\delta_{i f}(t)\right\|<2 \varepsilon_{f} \\
1, & \left\|\delta_{i f}(t)\right\| \geq 2 \varepsilon_{f}\end{cases}
\end{array}
$$

where $\varepsilon_{k}, \varepsilon_{f}>0$ are small design constants. In (III.2), via the switching term $\beta_{i}(t)$, the controller switches between a translational action (III.3) to satisfy $\left|A_{i} A_{j}\right| \cong d_{i j}$ and a rotational action (III.4) to move the agent $A_{i}$ towards $p_{i f}$, which can take place only when $\left|A_{i} A_{j}\right|$ is sufficiently close to $d_{i j}$.

In (III.4), $\bar{\delta}_{j i}^{\perp}(t)$ is the unit vector perpendicular to the distance vector $\delta_{j i}(t)=p_{j}(t)-p_{i}(t)$ with clockwise orientation with respect to the circle $C\left(p_{j}(t), d_{i j}\right)$, and the term $\operatorname{sgn}\left(\delta_{i f}(t)^{T} \bar{\delta}_{j i}^{\perp}(t)\right)$ determines the orientation of motion that would move $A_{i}$ towards $A_{i f}$. The switching term $\bar{\beta}_{i}(t)$ is for avoiding chattering due to small but acceptable errors in the final position of $A_{i}$.

\section{Control Law for the Leader}

Agent $A_{1}$ is the leader of the formation in Figure 3. The control law for $A_{1}$ is

$$
\begin{aligned}
& v_{i}(t)=\bar{v} \bar{\beta}_{i}(t) \delta_{i f}(t) /\left\|\delta_{i f}(t)\right\| \\
& \delta_{i f}(t)=p_{i f}-p_{i}(t) \\
& \bar{\beta}_{i}(t)= \begin{cases}0, & \left\|\delta_{i f}(t)\right\|<\varepsilon_{f} \\
\frac{\left|\delta_{i f}(t)\right|-\varepsilon_{f}}{\varepsilon_{f}} & \varepsilon_{f} \leq\left|\delta_{i f}(t)\right|<2 \varepsilon_{f} \\
1, & \left\|\delta_{i f}(t)\right\| \geq 2 \varepsilon_{f}\end{cases}
\end{aligned}
$$

Here, the switching term $\bar{\beta}_{i}(t)$ again prevents chattering due to small but acceptable errors in the final position of $A_{i}$.

\section{Obstacle Avoidance for Single Agents}

In [12], we have designed and analyzed obstacle avoiding motion control algorithms for motion of a single robotic agent in environments with a single obstacle as well as those with multiple obstacles, based on the notions of streamlines, vortex, sinks, and the Circle Theorem from fluid dynamics and designs provided in [13]. In this section, we summarise these control algorithms, use of which will be extended to formations of robots in the later sections. 


\section{A. Path Planning for the Single Agent-Single Obstacle Case}

The following motion control law has been provided in [12] for obstacle avoiding motion of a single robotic agent in an environment with a single obstacle:

$$
\begin{gathered}
v_{x i}=-C \beta_{i}(t) \frac{x_{i}}{x_{i}^{2}+y_{i}^{2}} \\
+C\left(\frac{R_{o 1}^{2}}{d_{o 1}^{2}}\right) \frac{x_{o 1} \bar{x}_{1}^{2}+R_{o 1}^{2} \bar{x}_{1}+\bar{y}_{1}\left(2 y_{o 1} x_{i}-x_{o 1}\left(y_{i}+y_{o 1}\right)\right)}{R_{o 1}^{4}+2 R_{o 1}^{2}\left(x_{o 1} \bar{x}_{1}+y_{o 1} \bar{y}_{1}\right)+\left(x_{o 1}^{2}+y_{o 1}^{2}\right) d_{o 1}^{2}}
\end{gathered}
$$

$$
\begin{gathered}
v_{y i}=-C \beta_{i}(t) \frac{y_{i}}{x_{i}^{2}+y_{i}^{2}} \\
+C\left(\frac{R_{o 1}^{2}}{d_{o 1}^{2}}\right) \frac{y_{o 1} \bar{y}_{1}^{2}+R_{o 1}^{2} \bar{y}_{1}+\bar{x}_{1}\left(2 x_{o 1} y_{i}-y_{o 1}\left(x_{i}+x_{o 1}\right)\right)}{R_{o 1}^{4}+2 R_{o 1}^{2}\left(x_{o 1} \bar{x}_{1}+y_{o 1} \bar{y}_{1}\right)+\left(x_{o 1}^{2}+y_{o 1}^{2}\right) d_{o 1}^{2}}
\end{gathered}
$$

where $d_{o i}=\left\|p_{i}-c_{o 1}\right\|, \bar{p}_{i}=\left[\bar{x}_{i}, \bar{y}_{i}\right]^{T}=p_{i}-c_{o 1}$ and $\beta_{i}(t)$ is a smoothing function

$$
\beta_{i}(t)= \begin{cases}0, & \delta_{i g}(t)<\varepsilon_{k} \\ \frac{\delta_{i g}(t)-\varepsilon_{k}}{\varepsilon_{k}} & \varepsilon_{k} \leq \delta_{i g}(t)<2 \varepsilon_{k} \\ 1, & \delta_{i g}(t) \geq 2 \varepsilon_{k}\end{cases}
$$

where $\delta_{i g}(t)=\left\|p_{i}(t)-p_{i f}\right\|$ and $\varepsilon_{k}$ is a small design constant which specifies how close the agent can get to the goal position, before it needs to stop. Note that $\left\|v_{i}(t)\right\|=C$ whenever $\beta_{i}(t)=1$.

\section{B. Path Planning for the Single Agent-Multiple Obstacle Case}

The following motion control law has been provided in [12] for obstacle avoiding motion of a single robotic agent in an environment with $N$ obstacles:

$$
v_{x i}=\sum_{j} \alpha_{j} v_{x j i}, \quad v_{y i}=\sum_{j} \alpha_{j} v_{y j i}
$$

where $\left[v_{x j i}, v_{y j i}\right]^{T}$ is the velocity field for agent $i$ generated by obstacle $j$ and,

$$
\alpha_{j}=\prod_{k \neq j} \frac{d_{k}}{d_{j}+d_{k}}
$$

where $d_{k}, d_{j}$ are distance functions.

\section{Path Generation and Motion Control of ELLIPSE FORMATION}

The streamlines are normally calculated at set, closely spaced points which are then plotted as a curve. It is possible to discretize the streamline points into a set of new waypoints, which are easier for agents to follow. The basic idea is that an agent moves from an initial (starting) position and visits all the waypoints in order to reach the final destination. For more details on the waypoint generation method see [12].

\section{A. Motion Control of the Agents in the Formation}

In the unicycle model of (II.3) with constant $\bar{v}_{i}(t)=C$, the only control input is the angular velocity $\omega_{i}(t)$ of the agent $i$. Let $v_{x d i}, v_{y d i}$ be the signals generated by (IV.1), (IV.2), setting $\beta_{i}(t)=1$ for all $t$ and replacing $v_{x i}$ and $v_{y i}$ with, respectively, $v_{x d i}$ and $v_{y d i}$, and define the desired velocity vector

$$
v_{d i}(t)=\left(v_{x d i}(t), v_{y d i}(t)\right)=\bar{v}_{d i}(t)\left(\cos \theta_{d i}(t), \sin \theta_{d i}(t)\right)
$$

Note that $\left\|v_{d i}(t)\right\|=C$ is constant in this case. It is assumed that the constant speed is set at

$$
\bar{v}_{i}(t)=C=\bar{v}_{d i}(t)
$$

Therefore, the velocity tracking error is given by

$$
\begin{aligned}
v_{e i}(t) & =v_{i}(t)-v_{d i}(t) \\
& =C\left(\cos \theta_{i}(t)-\cos \theta_{d i}(t), \sin \theta_{i}(t)-\sin \theta_{d i}(t)\right)
\end{aligned}
$$

We aim to drive $z_{1 i}(t)=\cos \theta_{i}(t)-\cos \theta_{d i}(t)$ and $z_{2 i}(t)=$ $\sin \theta_{i}(t)-\sin \theta_{d i}(t)$ to zero in order to drive $v_{e i}(t)$ to zero. A sufficient condition for convergence of $z_{1 i}(t), z_{2 i}(t)$ to zero is convergence of $z_{3 i}(t)=\theta_{i}(t)-\theta_{d i}(t)$ to zero. For this purpose, we use the following PI controller in generation of the angular velocity signal

$$
\begin{aligned}
\omega_{i}(t) & =\dot{\theta}_{i}(t) \\
& =K_{p}\left(\theta_{d i}(t)-\theta_{i}(t)\right)+K_{I} \int_{0}^{t}\left(\theta_{d i}(\tau)-\theta_{i}(\tau)\right) d \tau
\end{aligned}
$$

where $K_{p}>0$ is the proportional gain, and $K_{I}>0$ is the integral gain. Next we use a similar approach to derive linear control laws for the agents modeled with non-holonomic unicycle dynamics and non-constant speed.

\section{COHESIVE Motion OF Rigid Formations}

In this section we address Problem 1. The procedure for producing collision free paths, which a formation can follow, requires several steps. Most importantly, as shown in this Section, the fluid streamlines can be obtained via the methods in Section IV-A by use of the Circle Theorem. Next, more desirable paths are generated from the fluid streamlines using the waypoint selection method in Section V. This is then combined with the theory presented in Section II-D. Furthermore, non-holonomic unicycle dynamics are applied to each agent in this section with a PI controller, as in Section V-A.

\section{A. Formation Control Design}

1) Formations and Ellipses: The approach to path planning taken here is such that only one agent (the leader) follows a streamline (or the waypoints obtained from it), another agent is used to set the orientation of the formation (the first follower) and other agents act as ordinary followers. As a design requirement, the leader agent $A_{1}$ is assumed to 
be at the centre of the formation, which is bounded by an ellipse.

Remark 6.1: If there is a case that $A_{1}$ is not at the center of the formation, one will introduce a virtual leader agent $A_{0}$ at the center of the formation, and makes $A_{1}$ the first follower, and $A_{2}$ an ordinary follower.

As introduced in Section $\mathrm{V}$, to successfully avoid collision with obstacles, we have to determine buffer regions around the obstacles. The size of the buffer regions are related to the length of the semi-minor axis of the smallest ellipse which covers the agents in the formation.

2) Implementation of Control Laws: The control laws for a multi-agent formation with non-holonomic unicycle dynamics for each agent and leader-follower structure, are presented in the following.

To control the leader we use the control law given in (III.5), and for controlling the first follower the control law described in (III.2)-(III.4) is used. Control of ordinary followers is achieved by using (III.1).

The orientation of the formation is maintained by the first follower agent $A_{2}$. We find the minimum volume ellipse which surrounds the formation and then calculate its semimajor axis vector. Since the positions of the agent $A_{1}$ and $A_{2}$ are known by $A_{2}$, the vector between $A_{2}$ and $A_{1}$ is also known by $A_{2}$. The angle between these two vectors is used to ensure that, the semi-major axis of the ellipse (and hence the formation) is always aligned with line segments connecting waypoints. It is desired for this angle to be as close to zero as possible, which means that the formation has the correct orientation. In the control laws for the first follower $A_{2}$, in Section II-D, $\delta_{2 f}(t)$ of (III.3) changes to

$$
\delta_{2 f}(t)=p_{2 f}(t)-p_{2}(t)
$$

where $p_{2 f}(t)$ is given by

$$
p_{2 f}(t)=\left[x_{p}, y_{p}\right]^{T}+p_{1}(t)
$$

and $p_{2}(t)$ is the current position of the first follower, agent $A_{2}$. Moreover,

$$
\begin{aligned}
& x_{p}=\cos \bar{\theta} \Delta x_{12}(t)-\sin \bar{\theta} \Delta y_{12}(t) \\
& y_{p}=\sin \bar{\theta} \Delta x_{12}(t)+\cos \bar{\theta} \Delta y_{12}(t),
\end{aligned}
$$

where $\bar{\theta}$ is the desired angle between the edge connecting agent $A_{1}$ to $A_{2}$ and the line segment connecting the previous waypoint and the current waypoint. Furthermore, $\Delta_{12}(t)=$ $\left[\Delta x_{12}(t), \Delta y_{12}(t)\right]^{T}$ :

$$
\Delta_{12}(t)=d_{21} \frac{\delta_{w i}(t)}{\left\|\delta_{w i}(t)\right\|} .
$$

Where

$$
\delta_{w_{i}}(t)=w_{i+1}-p_{1}(t),
$$

and $p_{1}(t)$ is the current position of agent $A_{1}$.

Remark 6.2: For the case that $\delta_{w i}(t)=0, \Delta_{12}(t)=0$, $p_{2 f}(t)=p_{1}(t)$ and the two agents $\left(A_{1}\right.$ and $\left.A_{2}\right)$ would collide. However with our algorithm, the formation switches to waypoint $w_{i+1}$ when the leader (or virtual agent) reaches within $\epsilon_{f}$ (which is the chattering constant in (III.5)) of

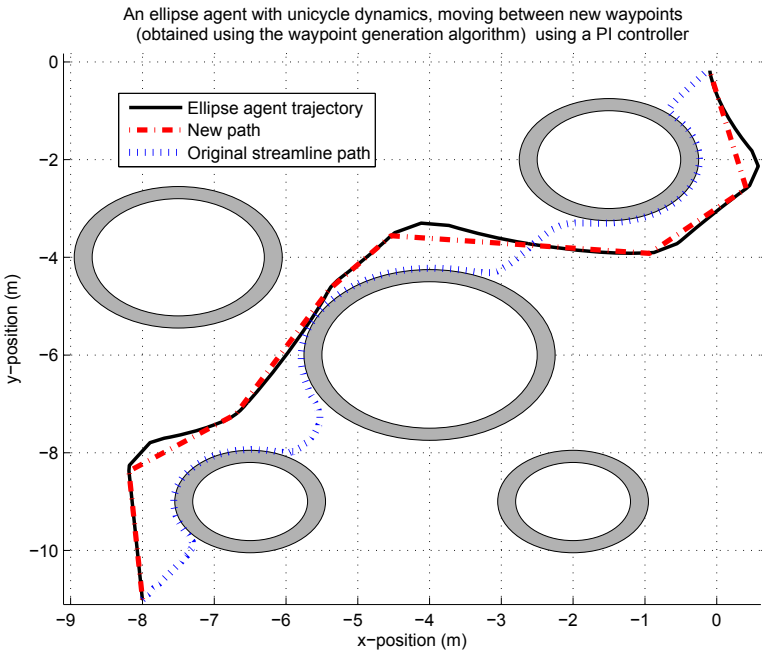

Fig. 4. Trajectory for an ellipse agent moving with unicycle dynamics, between new waypoints using the PI controller

waypoint $w_{i}$, provided that $\epsilon_{f}$ is less than the spacing between waypoints. It is because of this that $\delta_{w i}(t) \neq 0$ for all $t \geq 0$ in (VI.4).

\section{Simulations}

The formation shape that is used here for simulation purposes is shown in Figure 3. The trajectory of the ellipse formation simulated with unicycle dynamics, via PI control is shown in Figures 4. In this case, the ellipse parameters are $a_{m}=1, b_{m}=0.25$ and the angular velocity $\omega(t)$ has been limited to $\pm 1.5 \mathrm{rad} / \mathrm{s}$. Figure 5 shows the result when each agent in a five agent leader-follower formation is modeled with non-holonomic unicycle dynamics. In this simulation each agent has a constant speed of $1 \mathrm{~m} / \mathrm{s}$, the angular velocities $\omega_{i}(t)$ for agents were limited to $\pm 15 \mathrm{rad} / \mathrm{s}$ and the gains for all agents were $K_{p i}=10$ and $K_{I i}=5$, which were chosen arbitrarily. As can be seen, none of the agent trajectories have collided with the obstacles and there are no inter-agent collisions. Looking at the trajectories for the first and fourth followers it seems as though they deviate and get far from the formation at the waypoints. However the formation actually remains rigid and these deviations occur since in this particular simulation, the formation only rotates at each waypoint to align itself with the line segment between waypoints $w_{k}$ and $w_{k+1}$.

Figure 6 simulates the case where the formation continuously tries to align itself with the line segment connecting waypoints $w_{k}$ and $w_{k+1}$, rather than at each waypoint. Again, each agent is modeled with non-holonomic unicycle dynamics. In this simulation each agent has a constant speed of $1 \mathrm{~m} / \mathrm{s}$ except for the fifth agent (fourth follower) which has a speed of $1.3 \mathrm{~m} / \mathrm{s}$. Also, the angular velocities $\omega_{i}(t)$ for agents were limited to $\pm 10 \mathrm{rad} / \mathrm{s}$ and the gains for all agents were as above. This is a more desirable result than the simulation in Figure 5, but the drawback is that the fourth follower must have a larger speed than other the agents, in 


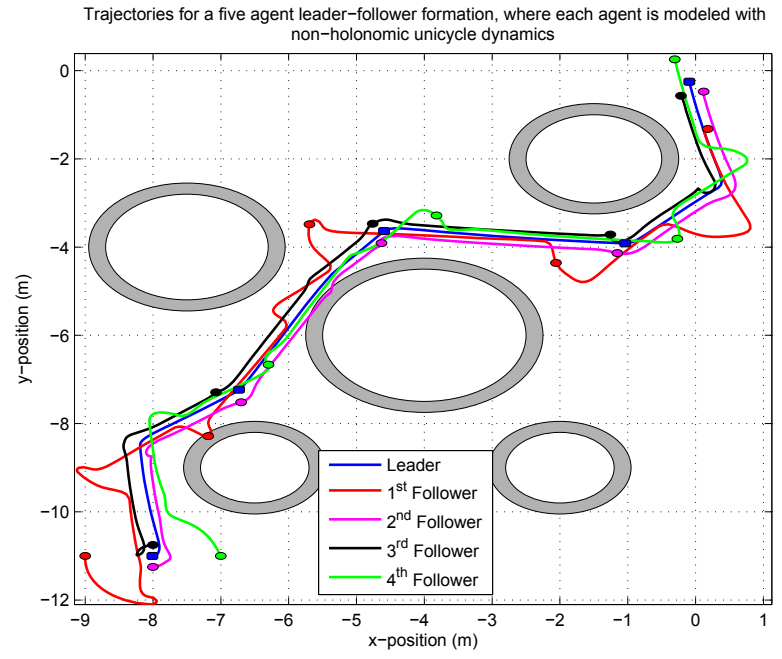

Fig. 5. Trajectories for a five agent leader-follower formation, where each agent is modeled with non-holonomic unicycle dynamics. Stop and go method.

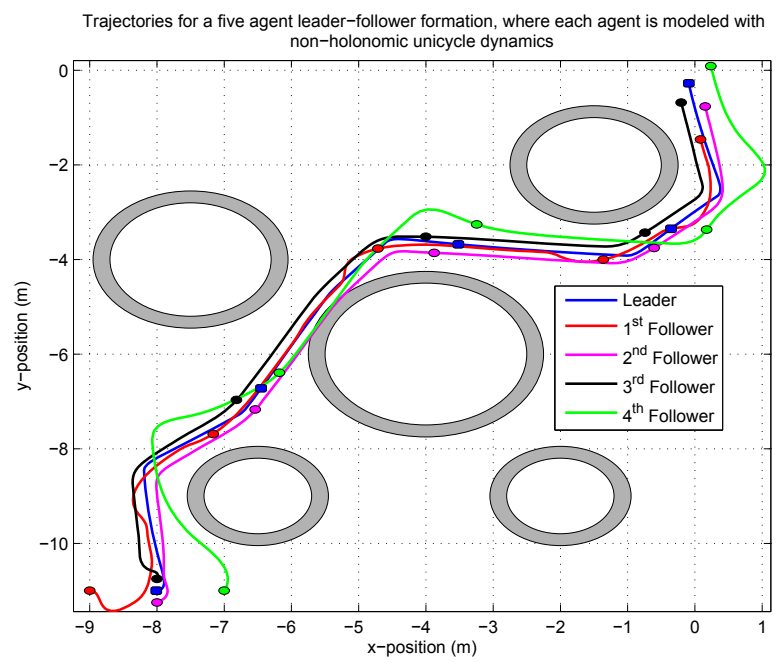

Fig. 6. Trajectories for a five agent leader-follower formation, where each agent is modeled with non-holonomic unicycle dynamics. Continuous motion.

order to properly maintain its distance constraints from the agents it follows. Again, the trajectory for the fourth follower seems to indicate that it deviates from the formation but it must do this in order for the formation to rotate and remain rigid.

\section{CONCLUDING REMARKS}

In this paper, we have proposed a method to produce collision free paths for swarms of agents moving in rigid formations, utilizing the Circle Theorem. The essence of these fluid mechanical principles is finding complex potentials for any given fictitious fluid flow region. From here stream functions are found and used to calculate the velocity field and streamlines of the flow region. The streamlines are used to visualize the fictitious fluid flow region. Furthermore, from these streamlines we calculate a set of waypoints which the leader of the formation (or the virtual leader at the centroid of the formation) must follow in order for the formation to avoid collision with obstacles. The agent models are considered to be non-holonomic unicycle dynamics. Proportional integral (PI) control is used for path following of the robots. The applicability of this approach is presented through successful simulation results.

One future research step is to provide a formal stability analysis to show that swarm agents converge to their desired paths, based on the proposed control techniques. Another future work topic is determining a way in which we can assign each robot agent of a formation to a fluid streamline, and implement a formation that can split and merge around obstacles, as required. Implementation of the Panel Method (or other approximate methods) to the formations under study, which allows the calculation of flow past multiple and much more complex obstacle geometries is also an important next step. Lastly, all of the control techniques presented in this paper, are based on pre-computed robot paths. A future study is on dynamic use of the same approach in a changing environment.

\section{REFERENCES}

[1] N. E. Leonard and E. Fiorelli, "Virtual leaders, artificial potentials, and coordinated control of groups," in Proc of the 40th IEEE Conference on Decision and Control, Orlando, FL, December 2001, pp. 29682973.

[2] Q. Hui and W. M. Haddad, "Distributed nonlinear control algorithms for network consensus," Automatica, vol. 44, no. 9, pp. 2375-2381, September 2008.

[3] N. Moshtagh and A. Jadbabaie, "Distributed geodesic control laws for flocking of nonholonomic agents," IEEE Transactions on Automatic Control, vol. 52, no. 4, pp. 681-686, April 2007.

[4] E. W. Justh and P. S. Krishnaprasad, "Equilibria and steering laws for planar formations," Systems \& Control Letters, vol. 52, no. 1, pp. 25-38, May 2004

[5] J. Sullivan, S. Waydo, and M. Campbell, "Using stream functions for complex behavior and path generation," in AIAA Guidance, Navigation and Control Conference, August 2003.

[6] I. Yavrucuk and O. Uzol, "Panel method based motion planning for swarming MAVs with probabilistic target tracking," in AIAA Guidance, Navigation and Control Conference and Exhibit, Hilton Head, South Carolina, August 2007.

[7] O. Uzol, I. Yavrucuk, and N. Sezer-Uzol, "Collaborative target tracking for swarming MAVs using potential fields and panel methods," in AIAA Guidance, Navigation and Control Conference and Exhibit, Honolulu, Hawaii, August 2008.

[8] B. Fidan, B. Anderson, C. Yu, and J. Hendrickx, In Modeling and Control of Complex Systems (P. Ioannou and A. Pitsillides (ed.)). Taylor \& Francis, 2007, ch. Persistent autonomous formations and cohesive motion control, pp. 247-275.

[9] V. Gazi and B. Fidan, In Swarm Robotics: SAB 2006 (ed. E. Sahin, W.M. Spears, and A.F.T. Winfield), ser. Lecture Notes in Computer Science. Springer-Verlag, 2007, ch. Coordination and control of multi-agent dynamic systems: Models and approaches, pp. 71-102.

[10] S. Sandeep, B. Fidan, and C. Yu, "Decentralized cohesive motion control of multi-agent formations," in In Proc. 14th Mediterranean Conference on Control and Automation, June 2006, pp. 1-6.

[11] I. Shames, C. Yu, B. Fidan, and B. Anderson, "Externally excited coordination of autonomous formations," in Mediterranean Conference on Control \& Automation, June 2007, pp. 1-6.

[12] R. Soukieh, I. Shames, and B. Fidan, "Obstacle avoidance of nonholonomic unicycle robots based on fluid mechanical modeling," in To appear in European Control Conference 2009, April 2009.

[13] S. Waydo, "Vehicle motion planning using stream functions," Control and Dynamical Systems, California Institute of Technology, CDS technical report, May 2003. 